\title{
Solutions of First-Order Volterra Type Linear Integrodifferential Equations by Collocation Method
}

\author{
Olumuyiwa A. Agbolade ${ }^{1,2}$ and Timothy A. Anake ${ }^{2}$ \\ ${ }^{1}$ Department of Mathematics and Statistics, Federal Polytechnic, Ilaro, Ogun State, Nigeria \\ ${ }^{2}$ Department of Mathematics, College of Science and Technology, Covenant University, Ota, Ogun State, Nigeria \\ Correspondence should be addressed to Timothy A. Anake; timothy.anake@covenantuniversity.edu.ng
}

Received 28 November 2016; Revised 22 February 2017; Accepted 2 March 2017; Published 20 March 2017

Academic Editor: Mehmet Sezer

Copyright (c) 2017 Olumuyiwa A. Agbolade and Timothy A. Anake. This is an open access article distributed under the Creative Commons Attribution License, which permits unrestricted use, distribution, and reproduction in any medium, provided the original work is properly cited.

\begin{abstract}
The numerical solutions of linear integrodifferential equations of Volterra type have been considered. Power series is used as the basis polynomial to approximate the solution of the problem. Furthermore, standard and Chebyshev-Gauss-Lobatto collocation points were, respectively, chosen to collocate the approximate solution. Numerical experiments are performed on some sample problems already solved by homotopy analysis method and finite difference methods. Comparison of the absolute error is obtained from the present method and those from aforementioned methods. It is also observed that the absolute errors obtained are very low establishing convergence and computational efficiency.
\end{abstract}

\section{Introduction}

Integrodifferential equation is a hybrid of integral and differential equations which have found extensive applications in sciences and engineering since it was established by Volterra [1]. A special class of these equations are the Volterra type which have been used to model heat and mass diffusion processes, biological species coexisting together with increasing and decreasing rate of growth, electromagnetic theory, and ocean circulations, among others [2].

First-order integrodifferential equation (IDE) of the Volterra type is generally of the form

$$
y^{\prime}=f(t, y(t), z(t)) \quad y\left(t_{0}\right)=y_{0},
$$

where

$$
z(t)=\int_{t_{0}}^{t} K(t, s, y(s)) d s, \quad t \in I .
$$

In solving (1), we seek the unknown function $y(t)$ given the kernel $K$, a nonsingular function defined on $S \times \mathbb{R}$ with $S:=$ $\left\{(t, s), t_{0} \leq s \leq t \leq T\right\}$. This kernel determines the nature of the solutions of integral equation (2) depending on its type
[3]. In this paper, only separable or degenerate kernels have been considered.

The theory and application of integrodifferential equations are important subjects in applied mathematics. The existence and uniqueness of the solutions of integrodifferential equations, usually discussed in terms of their kernel, had been established already in Linz [1]. Generally, methods for solving integrodifferential equations combine methods of solving both integral and differential equations. Also, since closed form solutions may not be tractable for most applications, numerical methods are employed to obtain approximations to the exact solutions.

Some numerical approaches in literature include iterative methods [4], successive approximation methods [5], and standard integral collocation approximation methods [6]. Other methods such as power series methods, where Chebyshev and Legendre's polynomials are used as basis functions, have been applied to obtain solutions of some higher order IDE of linear type. Akyaz and Sezer [7], for instance, presented Chebyshev collocation method for solving linear integrodifferential equations by truncated Chebyshev series. Recently, Gegele et al. [8] used power and Chebyshev series approximation methods to find numerical solution to higher 
order linear Fredholm integrodifferential equations using collocation methods. The result presented showed that the methods can give accurate results when compared with the exact solution. These methods proved efficient in the respective applications from the results provided but they seem yet to be applied to integrodifferential equations of Volterra type.

It is our aim here to extend the approach in Gegele et al. [8] to obtain approximate solutions for integrodifferential equations of Volterra type.

In the next section, we shall discuss the derivation of our methods; then the implementation using some sample problems is presented in Section 3. Finally, in Section 4 we shall present the results and draw our conclusions.

\section{Methodology}

In the sequel, the combination of the power series approximation and collocation method is employed for the solution of IDE of Volterra type.

To proceed, (1) is reduced to the form

$$
\begin{aligned}
y^{\prime}(x) & =F(x)+\int_{a}^{x} K(x, t) y(t) d t, \\
y^{(j)}(0) & =a_{j}, \quad j=0,1,
\end{aligned}
$$

where $y^{(j)}=d^{j} y / d x^{j}$ and $y^{(0)}=y$. The initial conditions (3b) are required in order to find particular solutions of (3a).

Now, let the solution $y(x)$ of Volterra type IDE, (3a) and (3b), be analytic and therefore possess the power series

$$
y(x)=\sum_{i=0}^{N} a_{i} x^{i}, \quad i \geq 0,
$$

where $x^{i}$ s are monomial bases and $a_{i}$ s are real coefficients to be determined.

Substituting equation (4) into both sides of (3a) gives

$$
\sum_{i=0}^{N} i a_{i} x^{(i-1)}=F(x)+\sum_{i=0}^{N} a_{i} \int_{a}^{x} t^{i} K(x, t) d t .
$$

Hence,

$$
F(x)=\sum_{i=0}^{N} a_{i}\left(i x^{(i-1)}-\int_{a}^{x} t^{i} K(x, t) d t\right),
$$

where $F(x)$ and $K(x, t)$ are known functions.

For an arbitrary choice of $N,(6)$ is obtained as a linear algebraic equation in $N+1$ unknowns as follows:

$$
\begin{aligned}
a_{0}+ & a_{1} \tau_{1}(x)+a_{2} \tau_{2}(x)+\cdots+a_{N-1} \tau_{N-1}(x) \\
& +a_{N} \tau_{N}\left(x_{1}\right)=F(x) .
\end{aligned}
$$

We note that $a_{0}$ is given by the initial condition (3b) while the remaining $a_{i}, i=1, \ldots, N$, are to be determined by collocation method.

To generate the collocation points, we shall consider two methods, namely, the standard and Chebyshev-GaussLobatto Collocation Methods, respectively.

2.1. Standard Collocation Method (SCM). This method is used to determine the desired collocation points within an interval, say, $[\vartheta, \sigma]$, and is given by

$$
x_{p}=\vartheta+\frac{(\sigma-\vartheta)}{N} p, \quad p=1,2,3, \ldots, N .
$$

2.2. Chebyshev-Gauss-Lobatto Collocation Method (CGLCM). The collocation points are obtained as follows:

$$
x_{p}=\cos \left(\frac{\pi p}{N}\right) \quad p=1,2,3, \ldots, N \text {. }
$$

Interestingly, Chebyshev-Gauss-Lobatto points have also been used as collocation and interpolation points in the solutions of optimal control problems governed by Volterra integrodifferential equations $[9,10]$.

Using either of the two collocation points to collocate (7) together with the initial conditions given in (3b) will result in a system of $N+1$ linear algebraic equations in $N+1$ unknowns. Hence, the resultant matrix problem is as follows:

$$
\left[\begin{array}{cccccc}
1 & 0 & 0 & \cdots & 0 & 0 \\
0 & \tau_{1}\left(x_{1}\right) & \tau_{2}\left(x_{1}\right) & \cdots & \tau_{N-1}\left(x_{1}\right) & \tau_{N}\left(x_{1}\right) \\
0 & \tau_{1}\left(x_{2}\right) & \tau_{2}\left(x_{2}\right) & \cdots & \tau_{N-1}\left(x_{2}\right) & \tau_{N}\left(x_{2}\right) \\
& \vdots & & \vdots & & \vdots \\
0 & \tau_{1}\left(x_{N-1}\right) & \tau_{2}\left(x_{N-1}\right) & \cdots & \tau_{N-1}\left(x_{N-1}\right) & \tau_{N}\left(x_{N-1}\right) \\
0 & \tau_{1}\left(x_{N}\right) & \tau_{2}\left(x_{N}\right) & \cdots & \tau_{N-1}\left(x_{N}\right) & \tau_{N}\left(x_{N}\right)
\end{array}\right]\left[\begin{array}{c}
a_{0} \\
a_{1} \\
a_{2} \\
\vdots \\
a_{N-1} \\
a_{N}
\end{array}\right]=\left[\begin{array}{c}
F\left(x_{0}\right) \\
F\left(x_{1}\right) \\
F\left(x_{2}\right) \\
\vdots \\
F\left(x_{N-1}\right) \\
F\left(x_{N}\right)
\end{array}\right]
$$

where $\tau_{j}\left(x_{p}\right), j=1,2,3, \ldots, N$, are polynomials evaluated at each collocation point $x_{p}$. The values of the unknowns can be obtained using any convenient method of solving matrix equations of the form $A X=B$, where $A$ is invertible.
Substituting the values of the $a_{i}, i=0,1,2, \ldots, N$, obtained from (4) yields the approximate solution. We note that the accuracy level desired for the approximate solution is determined by the degree of the approximating polynomial. 


\section{Results}

In this section, standard and Chebyshev-Gauss-Lobatto collocation points have been employed, respectively, to solve sample problems as described in Section 2. The numerical solutions obtained using the present method had been compared with the exact solutions of the sample problems. Similarly, absolute errors of results from this present method have been compared with those obtained in Behrouz [11] by homotopy analysis method (HAM) and finite difference method (FDM) for the same problems.

The absolute error of computation is defined in all cases as follows:

$$
\left|y\left(x_{i}\right)-Y\left(x_{i}\right)\right| \quad \vartheta \leq x_{i} \leq \sigma, i=1,2,3, \ldots .
$$

Problem 1.

$$
\begin{array}{r}
y^{\prime}(x)+y(x)=\left(x^{2}+2 x+1\right) e^{-x}+5 x^{2}+8 \\
-\int_{0}^{x} t y(t) d t, \quad y(0)=10 .
\end{array}
$$

Exact solution: $y(x)=10-x e^{-x}$.

Using SCM, we obtained the following approximate solutions:

$$
\begin{aligned}
y(x)= & 10-0.999955 x+0.999606 x^{2}-0.498312 x^{3} \\
& +0.162656 x^{4}-0.0362999 x^{5} \\
& +0.00442505 x^{6}
\end{aligned}
$$

Similarly using CGLCM, we obtained the approximate solution as follows:

$$
\begin{aligned}
y(x)= & 10-x+1.00115 x^{2}-0.499641 x^{3} \\
& +0.163495 x^{4}-0.0434569 x^{5}+0.0107202 x^{6} .
\end{aligned}
$$

The solutions obtained from the implementation of the method for Problem 1 using SCM and CGLCM are compared with the exact solution in Table 1. Also absolute errors obtained are compared with absolute errors obtained from HAM and FDM in Table 2.

Problem 2.

$$
y^{\prime}(x)+y(x)=\int_{0}^{x} e^{t-x} y(t) d t, \quad y(0)=1 .
$$

Exact solution: $y(x)=e^{-x} \cosh x$.

Using SCM, we obtained the following approximate solutions:

$$
\begin{aligned}
y(x)= & 1-0.999759 x+0.997930 x^{2}-0.658238 x^{3} \\
& +0.314202 x^{4}-0.107608 x^{5}+0.0235161 x^{6} \\
& -0.00237066 x^{7} .
\end{aligned}
$$

TABLE 1: Comparison of exact solution with numerical solutions for Problem 1.

\begin{tabular}{lccc}
\hline$x_{i}$ & Exact & SCM & CGLCM \\
\hline 0.0000 & 0.0000 & 0.0000 & 0.0000 \\
0.0714 & 9.933495516 & 9.933497240 & 9.933501428 \\
0.1429 & 9.876160300 & 9.876162226 & 9.876183411 \\
0.2143 & 9.827046197 & 9.827047975 & 9.827095298 \\
0.2857 & 9.785292202 & 9.785293839 & 9.785371414 \\
0.3571 & 9.750116951 & 9.750118464 & 9.750223918 \\
0.4286 & 9.720811832 & 9.720813173 & 9.720936698 \\
0.5000 & 9.696734670 & 9.696735770 & 9.696860288 \\
0.5714 & 9.677303930 & 9.677304767 & 9.677407809 \\
0.6429 & 9.661993413 & 9.661994038 & 9.662051938 \\
0.7143 & 9.650327386 & 9.650327889 & 9.650322899 \\
0.7857 & 9.641876128 & 9.641876560 & 9.641807476 \\
0.8571 & 9.636251847 & 9.636252142 & 9.636149063 \\
0.9286 & 9.633104936 & 9.633104922 & 9.633048727 \\
1.0000 & 9.632120559 & 9.632120150 & 9.632267300 \\
\hline
\end{tabular}

Similarly, using CGLCM we obtained the approximate solution as follows:

$$
\begin{aligned}
y(x)= & 1-0.99941 x+0.999958 x^{2}-0.671295 x^{3} \\
& +0.331381 x^{4}-0.123419 x^{5}+0.0496174 x^{6} \\
& -0.0193272 x^{7} .
\end{aligned}
$$

The solutions obtained from the implementation of the method for Problem 2 using SCM and CGLCM are compared with the exact solution in Table 3. Also absolute errors obtained are compared with absolute errors obtained from HAM and FDM in Table 4.

\section{Conclusion}

In this paper, numerical solution of Volterra type integrodifferential equation of first order with degenerate kernels is obtained by power series collocation method based on two collocating points methods, namely, Standard Collocation Method (SCM) and Chebyshev-Gauss-Lobatto Collocation Method (CGLCM), presented.

The two methods for selecting collocation points yielded different schemes from which approximate solutions were obtained, respectively, and compared with the exact solutions as shown in Tables 1 and 3 . From the results presented, the two methods gave good results for first-order integrodifferential equations of Volterra type.

The comparison of absolute errors of the results obtained by the present method with those by homotopy analysis method and finite difference method for the same problems revealed that the method is efficient and cheap for the numerical solutions of first-order integrodifferential equation of Volterra type as illustrated in Tables 2 and 4 . The performance of the present method against homotopy analysis method is expected as the latter is a semianalytic method. 
TABLE 2: Comparison of absolute errors for Problem 1

\begin{tabular}{lcccc}
\hline$x_{i}$ & SCM & CGLCM & FDM & HPM \\
\hline 0.0000 & 0.0000 & 0.0000 & 0.0000 & 0.0000 \\
0.0714 & $1.72431 E-06$ & $5.91262 E-06$ & $2.85397 E-04$ & $5.15735 E-07$ \\
0.1429 & $1.92637 E-06$ & $2.31105 E-05$ & $2.98284 E-04$ & $3.00036 E-07$ \\
0.2143 & $1.77825 E-06$ & $4.91013 E-05$ & $5.43393 E-04$ & $2.80293 E-06$ \\
0.2857 & $1.63695 E-06$ & $7.92123 E-05$ & $5.11413 E-04$ & $1.47980 E-05$ \\
0.3571 & $1.51288 E-06$ & $1.06967 E-04$ & $7.15638 E-04$ & $4.60491 E-05$ \\
0.4286 & $1.34028 E-06$ & $1.24865 E-04$ & $6.54200 E-04$ & $1.11168 E-04$ \\
0.5000 & $1.09939 E-06$ & $1.25617 E-04$ & $8.18261 E-04$ & $2.40330 E-04$ \\
0.5714 & $8.36590 E-07$ & $1.03879 E-04$ & $7.38321 E-04$ & $4.73070 E-04$ \\
0.6429 & $6.24770 E-07$ & $5.85256 E-05$ & $7.73248 E-04$ & $8.52587 E-04$ \\
0.7143 & $5.03018 E-07$ & $4.48751 E-06$ & $8.63249 E-04$ & $1.45361 E-03$ \\
0.7857 & $4.31615 E-07$ & $6.86526 E-05$ & $7.66939 E-04$ & $2.36487 E-03$ \\
0.8571 & $2.95402 E-07$ & $1.02783 E-04$ & $8.24573 E-04$ & $3.71115 E-03$ \\
0.9286 & $1.39661 E-08$ & $5.62088 E-05$ & $7.26353 E-04$ & $5.63206 E-04$ \\
1.0000 & $4.08829 E-07$ & $1.46741 E-04$ & $8.32344 E-04$ \\
\hline
\end{tabular}

TABLE 3: Comparison of exact solution with numerical solution for Problem 2.

\begin{tabular}{lccc}
\hline$x_{i}$ & Exact & SCM & CGLCM \\
\hline 0.0000 & 0.0000 & 0.0000 & 0.0000 \\
0.0833 & 0.9232408624 & 0.9232506225 & 0.923287006 \\
0.1667 & 0.8582656553 & 0.8582754765 & 0.858341248 \\
0.2500 & 0.8032653299 & 0.8032737684 & 0.803340755 \\
0.3333 & 0.7567085595 & 0.7567163057 & 0.756749451 \\
0.4167 & 0.7172991043 & 0.7173066673 & 0.717278486 \\
0.5000 & 0.6839397206 & 0.6839470433 & 0.683851372 \\
0.5833 & 0.6557016120 & 0.6557084119 & 0.655570212 \\
0.6667 & 0.6317985691 & 0.6318047191 & 0.631680300 \\
0.7500 & 0.6115650801 & 0.6115707282 & 0.611530371 \\
0.8333 & 0.5944378014 & 0.5944432050 & 0.594525790 \\
0.9167 & 0.5799398730 & 0.5799451069 & 0.580071957 \\
1.0000 & 0.5676676416 & 0.5676724400 & 0.567505200 \\
\hline
\end{tabular}

TABLE 4: Comparison of absolute errors for Problem 2.

\begin{tabular}{lcccc}
\hline$x_{i}$ & SCM & CGLC & FDM & HPM \\
\hline 0.0000 & 0.0000 & 0.0000 & 0.0000 & 0.0000 \\
0.0833 & $9.76008 E-06$ & $4.61438 E-05$ & $1.77203 E-02$ & $1.85469 E-09$ \\
0.1667 & $9.82124 E-06$ & $7.55931 E-05$ & $2.16887 E-03$ & $3.13105 E-10$ \\
0.2500 & $8.43856 E-06$ & $7.54254 E-05$ & $1.89273 E-03$ & $1.14368 E-09$ \\
0.3333 & $7.74622 E-06$ & $4.08918 E-05$ & $4.52374 E-03$ & $8.37039 E-11$ \\
0.4167 & $7.56304 E-06$ & $2.06182 E-05$ & $2.06181 E-02$ & $2.65354 E-09$ \\
0.5000 & $7.32270 E-06$ & $8.83487 E-05$ & $7.13624 E-03$ & $3.14279 E-10$ \\
0.5833 & $6.79994 E-06$ & $1.31400 E-05$ & $1.10585 E-02$ & $1.24270 E-09$ \\
0.6667 & $6.15006 E-06$ & $1.18269 E-05$ & $8.20866 E-03$ & $5.57863 E-10$ \\
0.7500 & $5.64809 E-06$ & $3.47095 E-05$ & $3.41335 E-03$ & $1.32579 E-09$ \\
0.8333 & $5.40361 E-06$ & $8.79889 E-05$ & $8.16328 E-03$ & $6.81219 E-10$ \\
0.9167 & $5.23390 E-06$ & $1.32084 E-05$ & $2.89396 E-03$ & $5.16015 E-09$ \\
1.0000 & $4.79838 E-06$ & $1.62442 E-05$ & $3.27168 E-03$ & $9.48169 E-09$ \\
\hline
\end{tabular}




\section{Conflicts of Interest}

The authors declare that there are no conflicts of interest regarding the publication of this paper.

\section{Acknowledgments}

The authors are grateful to the Covenant University Centre for Research, Innovation and Development (CUCRID) for sponsoring this publication.

\section{References}

[1] P. Linz, Analytic and Numerical Methods for Volterra Equations, SIAM, Philadelphia, Pa, USA, 1985.

[2] M. Rahman, Integral Equations and Their Applications, WIT Press, Southampton, UK, 2007.

[3] G. Tiwari, "Solving Integral Equations: The Interactive Way!," 2014, https://gauravtiwari.org/.

[4] A. J. Jerri, Introduction to Integral Equations with Applications, Marcel Dekken, Inc, New York, NY, USA, 1985.

[5] R. K. Saeed, Computational methods for solving system of linear Volterra integral and integro-differential equation [Ph.D. thesis], University of Salahaddin Hawler, College of Science, 2006.

[6] A. Abubakar and O. A. Taiwo, "Integral collocation approximation methods for the numerical solution of high-orders linear Fredholm-Volterra integro-differential equations," American Journal of Computational and Applied Mathematics, vol. 4, no. 4, pp. 111-117, 2014.

[7] A. Akyaz and M. Sezer, "A Chebychev collocation method for the solution of linear integro-differential equation," International Journal of Computer Mathematics, vol. 72, no. 4, pp. 491507, 1999.

[8] O. A. Gegele, O. P. Evans, and D. Akoh, "Numerical solution of higher order linear Fredholm-integro-differential equations," American Journal of Engineering Research, vol. 8, no. 3, pp. 243247, 2014.

[9] M. El-Kady and H. Moussa, "Monic Chebyshev approximations for solving optimal control problem with Volterra integro differential equations," General Mathematics Notes, vol. 14, no. 2, pp. 23-36, 2013.

[10] W. Zhang and H. Ma, "The Chebyshev-Legendre collocation method for a class of optimal control problems," International Journal of Computer Mathematics, vol. 85, no. 2, pp. 225-240, 2008.

[11] R. Behrouz, "Numerical solutions of the linear Volterra integrodifferential equations: homotopy perturbation method and finite difference method," World Applied Sciences Journal, vol. 9, pp. 7-12, 2010. 


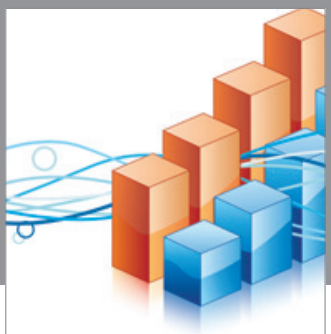

Advances in

Operations Research

vatem alat4

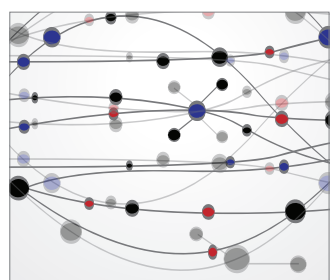

\section{The Scientific} World Journal
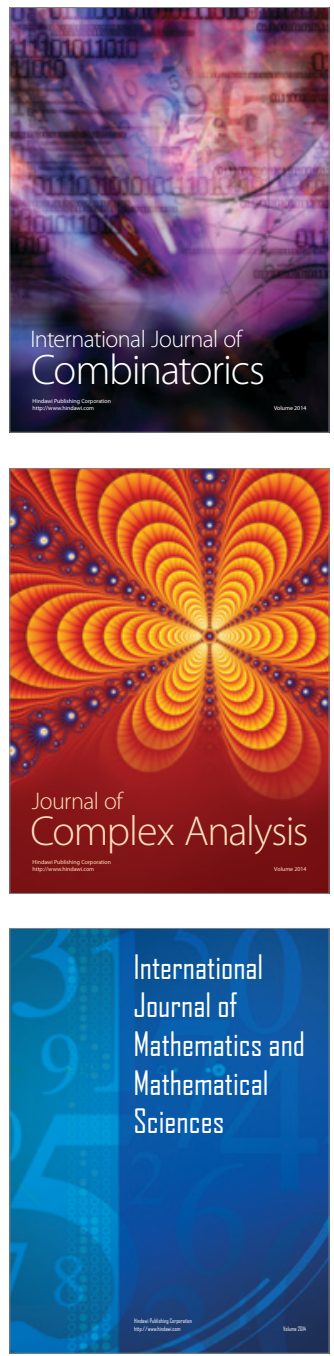
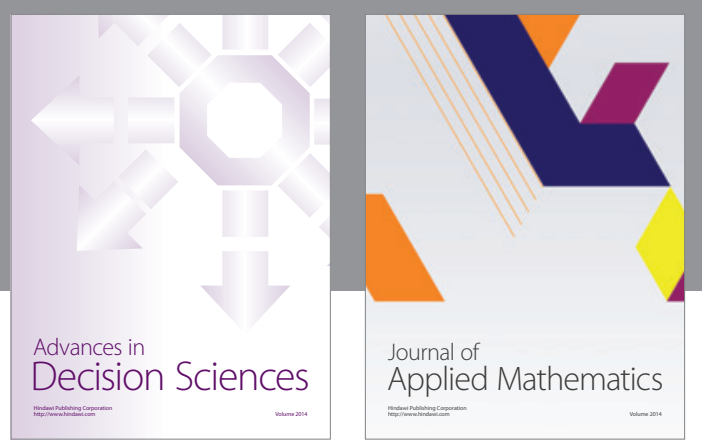

Algebra

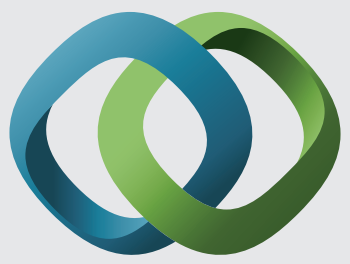

\section{Hindawi}

Submit your manuscripts at

https://www.hindawi.com
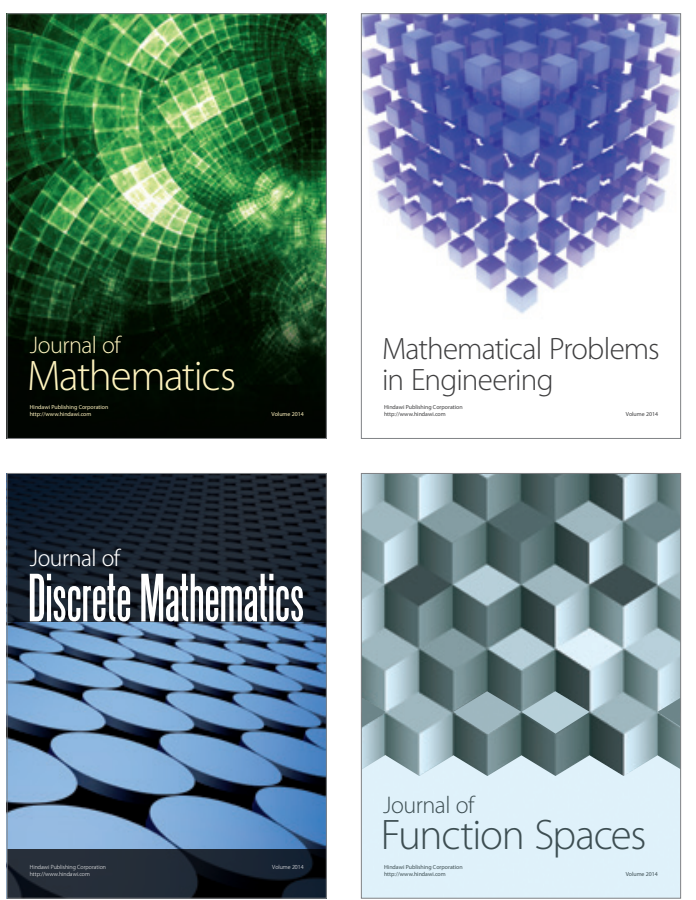

Mathematical Problems in Engineering
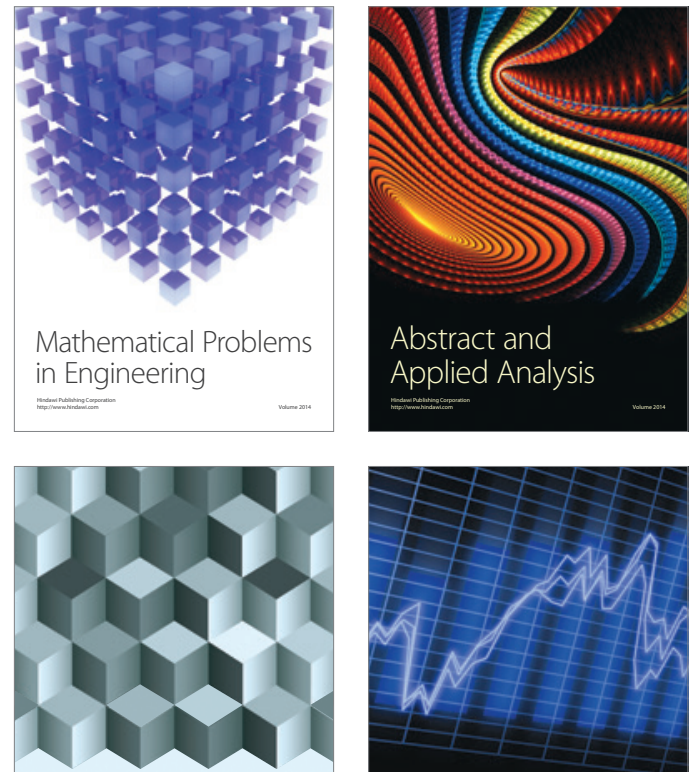

Journal of

Function Spaces

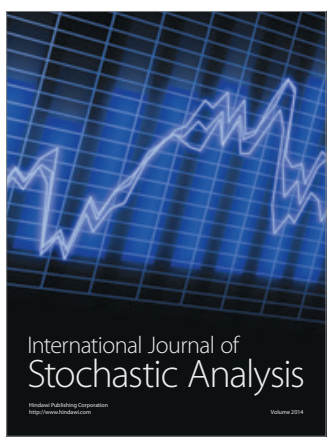

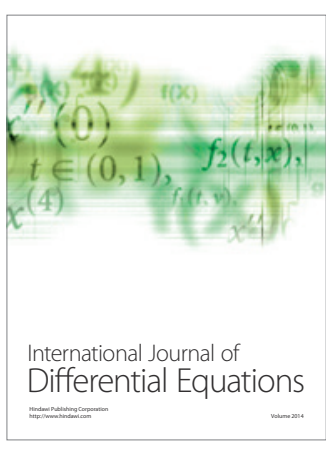
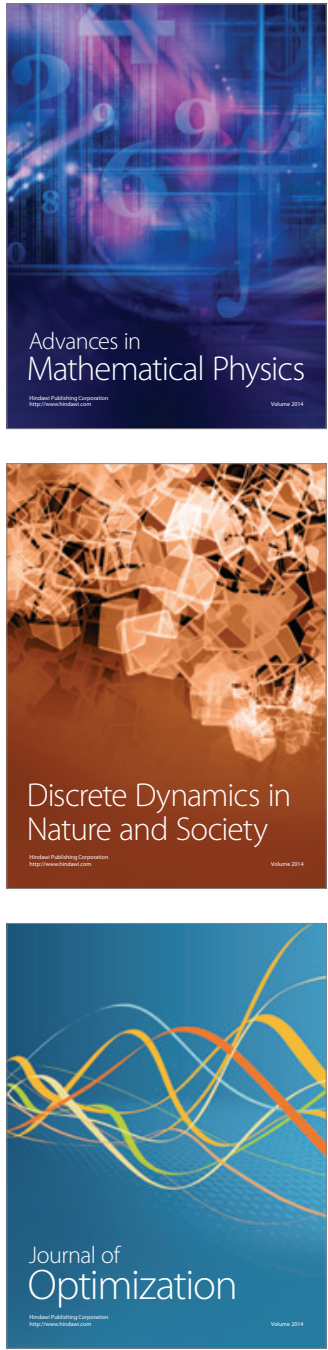